INPLASY

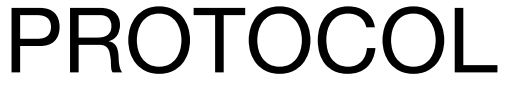

To cite: Chen et al.

Thermotherapy for shoulder pain: a protocol for systematic review. Inplasy protocol

2021110086. doi:

10.37766/inplasy2021.11.0086

Received: 23 November 2021

Published: 23 November 2021

Corresponding author:

Shen Cimin

shencimin@163.com

Author Affiliation:

Department of Acupuncture and Moxibustion, Fenghua Hospital of Traditional Chinese Medicine, Ningbo, Zhejiang Province.

Support: The Basic scientific research.

Review Stage at time of this submission: Piloting of the study selection process.

Conflicts of interest:

None declared.

\section{Thermotherapy for shoulder pain: a protocol for systematic review}

Chen, B1; Shen, CM²; Li, N3; Wang, L4; Chen, DD5.

Review question / Objective: Shoulder pain is a common musculoskeletal disorder prompting many patients to seek treatment. Thermotherapy is a common treatment for shoulder which has been widely used in hospitals. But its efficiency has not been scientifically and methodically evaluated. This protocol aims to evaluate the efficacy and safety of Thermotherapy for treating shoulder pain.

Information sources: Eight databases will be searched from their inception to October 2021. They are as follows: PubMed, Embase, Cochrane Library, ClinicalTrials.gov, China Knowledge Resource Integrated Database (CNKI), Weipu Database for Chinese Technical Periodicals (VIP), Chinese Biomedical Literature Database (CBM), and Wanfang Database. There will be no limitation to study publication status or language. The search terms include shoulder impingement syndrome, rotator cuff, bursitis, adhesive capsulitis, frozen shoulder, shoulder pain, thermotherapy, diathermy, heat therapy, Moxibustion, and RCTs. The equivalent search words will be used in the Chinese databases.

INPLASY registration number: This protocol was registered with the International Platform of Registered Systematic Review and Meta-Analysis Protocols (INPLASY) on 23 November 2021 and was last updated on 23 November 2021 (registration number INPLASY2021110086).

\section{INTRODUCTION}

Review question / Objective: Shoulder pain is a common musculoskeletal disorder prompting many patients to seek treatment. Thermotherapy is a common treatment for shoulder which has been widely used in hospitals. But its efficiency has not been scientifically and methodically evaluated. This protocol aims to evaluate the efficacy and safety of Thermotherapy for treating shoulder pain.

Condition being studied: In a US survey, musculoskeletal conditions were reported by $50 \%$ of adults. Indeed, a high prevalence of shoulder pain is experienced by over one-quarter of the general population in 
their lifetimes. In China, the incidence of women over 40 is $45 \%$, and the incidence is $8 \%$ for urban population. Unlike more prevalent conditions such as low back pain, with a low risk of chronicity (ie, persisting $\geq 12$ weeks), nontraumatic musculoskeletal shoulder pain persists beyond 6 months in $50 \%$ of individuals. It is a disabling condition that negatively impacts quality of life, psychological wellbeing, and the ability to maintain employment. There are many related conservation treatment applied on shoulder pain, such as corticosteroids, nonsteroidal anti-inflammatory drugs, acupuncture, manual therapy, and other physical therapy. And the effectiveness of most intervention is not definite. Thermotherapy is the application of heat to the body resulting in increased tissue temperature, Techniques for thermotherapy include the application of Moxibustion, hot packs, superficial heat, and via diathermy (application of electromagnetic energy). Thermotherapy is used in rehabilitation to reduce pain and stiffness, and to increase mobility. Thermotherapy helps to relax muscles and increase circulation to the affected area, thus reducing pain and stiffness, although there is some concern that this may, in turn, worsen inflammation and edema. Thermotherapy can be self applied easily by the patient at home(such as the use of heat packs), and may also be combined with other rehabilitation interventions. However, no systematic review has been performed to evaluate the effectiveness and safety of thermotherapy for shoulder pain. Therefore, the systematic review will assess the efficacy and safety of thermotherapy for shoulder pain.

\section{METHODS}

Participant or population: People with shoulder pain regardless of sex, age, or severity and duration of disease.

Intervention: Interventions using thermotherapy only were included in this review.

Comparator: Trials that compared thermotherapy with standard treatment and/or placebo were included. thermotherapy with another active therapy versus the same therapy alone will also be investigated. Trials comparing head to head therapies, such as two different types of diathermy, were not included in this review.

Study designs to be included: All RCTs of thermotherapy for shoulder pain without publication status restriction or writing language. Non-RCTs, quasi-RCTs, uncontrolled trials, reviews, casecontrolled studies, animal trials, and laboratory studies will be excluded.

Eligibility criteria: For continuous data, a mean difference or standardized mean difference with $95 \%$ confidence intervals will be applied. For dichotomous outcome data, the risk ratio with $95 \%$ confidence intervals will be used to evaluate the treatment effect.

Information sources: Eight databases will be searched from their inception to October 2021. They are as follows: PubMed, Embase, Cochrane Library, ClinicalTrials.gov, China Knowledge Resource Integrated Database (CNKI), Weipu Database for Chinese Technical Periodicals (VIP), Chinese Biomedical Literature Database (CBM), and Wanfang Database. There will be no limitation to study publication status or language. The search terms include shoulder impingement syndrome, rotator cuff, bursitis, adhesive capsulitis, frozen shoulder, shoulder pain, thermotherapy, diathermy, heat therapy, Moxibustion, and RCTs. The equivalent search words will be used in the Chinese databases.

Main outcome(s): The primary outcome of shoulder pain symptom is visual analog scale (0-10), the ability assessment of daily living activities. Adverse events incidence and shoulder range of motion will be accepted as the secondary outcomes.

Quality assessment / Risk of bias analysis: We will assess clinical and methodological diversity in terms of participants, interventions, outcomes and study 
characteristics for the included studies, to determine whether a meta-analysis is appropriate. When meta-analysis is appropriate, we will assess and quantify the possible magnitude of inconsistency (i.e. heterogeneity) across studies, using the 12 statistic with a guide for interpretation as follows: $0 \%$ to $40 \%$ might not be important; $30 \%$ to $60 \%$ may represent moderate heterogeneity; $50 \%$ to $90 \%$ may represent substantial heterogeneity; and $75 \%$ to $100 \%$ represents considerable heterogeneity.

Strategy of data synthesis: We plan to pool outcomes from trials with similar characteristics (participants, interventions and common comparators, outcome measures and timing of outcome measurement) to provide estimates of benefit and harm. We plan to synthesize effect estimates using a random-effects meta-analysis model based on the assumption that clinical diversity is likely to exist, and that different studies are estimating different intervention effects. Where we cannot pool data, we plan to present effect estimates and $95 \% \mathrm{Cls}$ of each trial in tables, and summarize the results in text.

Subgroup analysis: If we identify cases of considerable heterogeneity (defined as 12 of $75 \%$ or greater), we will explore the data further by comparing the characteristics of individual studies and performing subgroup analyses.

Sensitivity analysis: Sensitivity analysis. When there are sufficient studies, sensitivity analysis will be performed to assess the robustness of studies according to methodological quality, sample size, and missing data.

Country(ies) involved: China.

Keywords: shoulder pain, protocol, systematic review, thermotherapy.

Contributions of each author:

Author 1 - Chen Bin.

Author 2 - Shen Cimin.

Author 3 - Li Na.
Author 4 - Wang Lu.

Author 5 - Chen Dangdang. 